\title{
L'argomentazione riflessiva come strumento a supporto dei processi di valutazione formativa: il ruolo fondamentale del docente
}

\section{Reflective argumentation as a tool to support formative assessment processes: the key-role of the teacher}

\author{
Annalisa Cusi \\ Dipartimento di Matematica, Sapienza Università di Roma - Italia \\ 凶annalisa.cusi@uniroma1.it
}

Sunto / In questo articolo vengono proposte alcune riflessioni sul ruolo che il docente svolge nell'implementare pratiche mirate a valorizzare i processi di argomentazione riflessiva a supporto della valutazione formativa in matematica. Mediante l'analisi di alcuni stralci di discussione, tratti da una sperimentazione condotta in una classe seconda di una scuola secondaria di primo grado, si mettono in luce gli interventi chiave che il docente può proporre per stimolare processi di argomentazione riflessiva e attivare specifiche strategie di valutazione formativa.

Parole chiave: valutazione formativa; argomentazione riflessiva; discussioni di classe; ruolo dell’insegnante.

\begin{abstract}
This paper proposes some reflections on the role played by the teacher in implementing teaching practices aimed at promoting processes of reflective argumentation as a support for formative assessment in Mathematics. Through the analysis of some excerpts from a classroom discussion realized during a teaching experiment with lower secondary school students (grade 7), the teacher's key-interventions are highlighted, focusing on their role in stimulating reflective argumentation and in fostering the activation of specific formative assessment strategies.
\end{abstract}

Keywords: formative assessment; reflective argumentation; classroom discussions; role of the teacher.

\section{Introduzione}

In questo articolo si focalizzerà l'attenzione sui processi di valutazione formativa, riflettendo sul ruolo chiave che gli approcci che caratterizzano quella che Hoffmann (2016) definisce argomentazione riflessiva (reflective argumentation) possono svolgere nel favorire l'attivazione di specifiche strategie per la valutazione formativa (Black \& Wiliam, 2009). In particolare, attraverso l'analisi di alcuni stralci tratti da una discussione condotta nell'ambito di una sperimentazione, si rifletterà su quali interventi del docente possano risultare maggiormente efficaci nel supportare la creazione di un contesto di insegnamento-apprendimento nel quale si sviluppino ricchi processi di argomentazione riflessiva e si attivino molteplici strategie di valutazione formativa. 
In questo paragrafo vengono presentati gli aspetti teorici che fanno da sfondo alla ricerca oggetto di questo articolo:

1. la valutazione formativa in matematica, che permette di caratterizzare la metodologia didattica adottata durante la sperimentazione;

2. I'idea di reflective argumentation, inserita nel più ampio quadro riguardante i processi argomentativi in matematica;

3. il costrutto $M-C A_{C E}$ (acronimo per "Modello di comportamenti ed atteggiamenti consapevoli ed efficaci"), che consente di caratterizzare i ruoli attivati consapevolmente dall'insegnante durante le discussioni di classe.

Tali elementi forniscono anche le lenti teoriche per l'analisi dei dati raccolti durante la sperimentazione.

\title{
2.1 La valutazione formativa in matematica
}

Il ruolo formativo dei processi di valutazione nell'ambito dell'insegnamento-apprendimento della matematica - e dell'educazione scientifica più in generale - è stato valorizzato, in particolare nell'ultimo decennio, grazie alla realizzazione di diversi progetti di ricerca a livello internazionale (tra gli altri, si segnalano Bernholt, Ronnebeck, Ropohl, Koller \& Parchmann, 2013; Cusi, Morselli \& Sabena, 2017a; Ferretti, Paraskevi \& Vannini, 2018).

Secondo Black e Wiliam (2009), la pratica didattica è "formativa" nel momento in cui

\begin{abstract}
«[... ] elementi di evidenza relativi all'apprendimento degli studenti vengono raccolti, interpretati ed utilizzati da insegnanti, studenti e loro pari, per prendere decisioni sui passi successivi nel processo di istruzione, che possano essere migliori o meglio fondate, rispetto alle decisioni prese in assenza di tali elementi di evidenza».
\end{abstract}

(Black \& Wiliam, 2009, p. 7; traduzione dell'autrice)

Questa definizione mette in evidenza diversi aspetti della valutazione formativa, che si configura come vero e proprio metodo di insegnamento:

1. la valutazione formativa non si limita al momento della prova (scritta o orale), ma riguarda qualsiasi attività realizzabile nell'ambito dei processi di insegnamento-apprendimento;

2. I'insegnante non è il solo responsabile della realizzazione dei processi di valutazione formativa, poiché anche i singoli studenti e i loro pari sono protagonisti di tali processi;

3. le decisioni prese dai diversi attori coinvolti nei processi di valutazione formativa si basano sulle evidenze raccolte grazie a tali processi e ne rappresentano il principale obiettivo.

Nel caratterizzare le pratiche di valutazione formativa, Black e Wiliam (2009) identificano cinque strategie chiave, attivate dai diversi protagonisti del processo di valutazione (insegnante, studente e suoi pari):

Strategia 1. Chiarire/capire/condividere gli obiettivi di apprendimento e i criteri di valutazione (strategia attivata sia dall'insegnante, che dal singolo studente, che dai suoi pari);

Strategia 2. Progettare discussioni di classe efficaci e attività che consentano di mettere in luce l'apprendimento degli studenti (strategia attivata dall'insegnante); 
Strategia 3. Fornire feedback che consentano allo studente di migliorare (strategia attivata sia dall'insegnante che dai pari);

Strategia 4. Attivare gli studenti come risorse gli uni per gli altri (strategia attivata sia dall'insegnante, che dai pari);

Strategia 5. Attivare gli studenti come responsabili del proprio apprendimento (strategia attivata sia dall'insegnante, che dal singolo studente).

Fornire feedback rappresenta, in particolare, un aspetto centrale nell'ambito dei processi di valutazione formativa, poiché consente agli studenti di diventare maggiormente consapevoli degli obiettivi dei percorsi di insegnamento-apprendimento nei quali sono coinvolti, di monitorare i propri progressi verso tali obiettivi e di capire quali strategie attivare per superare le proprie difficoltà e progredire verso gli obiettivi prefissati.

Nell'ambito del progetto FaSMEd (Cusi, Morselli \& Sabena, 2017a, 2017b), è stata messa in luce l'incidenza dei fattori metacognitivi sui processi di valutazione formativa, mostrando l'efficacia di attività mirate a rendere visibile il pensiero degli studenti (Collins, Brown \& Newmann, 1989), attraverso la condivisione dei processi di pensiero con l'insegnante e con i compagni, e a stimolare una continua riflessione su tali processi. In particolare, è stato evidenziato come la progettazione di attività ad alto contenuto argomentativo, focalizzate sulla richiesta costante di motivare le risposte fornite, possa stimolare l'attivazione di processi metacognitivi, favorendo, da un lato, lo sviluppo di maggiori consapevolezze da parte degli studenti circa i propri processi di ragionamento, e, dall'altro, I'esplicitazione e conseguente condivisione dei loro processi di pensiero.

In questo modo, gli studenti hanno l'opportunità di sviluppare la capacità di riflettere approfonditamente sul proprio modo di affrontare le attività nelle quali sono coinvolti, divenendo così maggiormente responsabili del proprio apprendimento. Contemporaneamente, questo approccio fa sì che gli studenti sviluppino un maggior senso critico, cosa che permette loro di confrontarsi efficacemente con altri modi di ragionare e di affrontare le attività e i problemi matematici.

Il ruolo dell'argomentazione come strumento per supportare la valutazione formativa, attraverso I'attivazione di processi riflessivi a livello metacognitivo, viene approfondito nel prossimo paragrafo. Nell'ambito del progetto FaSMEd è stato, inoltre, studiato il ruolo dell'argomentazione come oggetto dei processi di valutazione formativa. In particolare, è stata evidenziata l'importanza di esplicitare e condividere con gli studenti alcuni criteri per valutare le argomentazioni da essi costruite (Cusi, Morselli \& Sabena, 2017b). Il primo criterio, quello della correttezza, focalizza l'attenzione sulla presenza o meno di errori di tipo matematico nella risposta costruita dagli studenti e nella giustificazione da essi fornita. II secondo criterio, quello della chiarezza, pone I'accento sulla comprensibilità della risposta da parte di un interlocutore (i compagni, l'insegnante), evidenziando l'importanza del piano comunicativo. II terzo criterio riguarda la completezza della risposta e richiede di verificare se i vari passaggi che conducono alla conclusione dell'argomento proposto sono stati esplicitati, facendo riferimento alle conoscenze matematiche coinvolte.

\subsection{Il ruolo chiave dell'argomentazione riflessiva}

Il ruolo cruciale dello sviluppo di competenze argomentative come parte fondamentale per lo sviluppo del pensiero matematico è stato il focus di diversi progetti di ricerca e formazione, come il progetto "Comunicazione e apprendimento" di Radford e Demers (2006), i progetti "Bambini Maestri Realtà" e "Linguaggio e argomentazione" del DIMA-Università degli Studi di Genova ${ }^{1}$ e il progetto Avimes-Piemonte (De Luca, Demartini, Migliano, Savioli, Serratore \& Vio, 2008).

1. Alcuni dei materiali progettati ed implementati nell'ambito di questo progetto sono reperibili al link http://pls.dima.unige.it/ azione1/argomentazione/azione1_argomentazione.php. 
L'idea che lo sviluppo di competenze argomentative supporti anche quello del pensiero matematico è in linea con l'idea di reflective argumentation introdotta da Hoffman (2016), che evidenzia come l'argomentazione possa rappresentare uno strumento per stimolare la riflessione sul proprio modo di ragionare, consentendo l'identificazione di punti deboli e punti di forza dei ragionamenti sviluppati, e una conseguente riformulazione delle idee e delle strategie alla base di tali ragionamenti.

Hoffmann definisce l'argomentazione riflessiva come:

«[...] un processo in cui la costruzione di argomenti viene utilizzata, intenzionalmente o non intenzionalmente, per stimolare uno o più dei seguenti processi: la riflessione sulla qualità dei propri argomenti; la riflessione sulla qualità del proprio modo di ragionare (il processo e il suo contenuto); I'identificazione di motivazioni per migliorare la qualità dei propri argomenti e ragionamenti; la ricerca creativa di modi per realizzare questo miglioramento; o la realizzazione di un cambiamento di prospettiva su uno di questi aspetti».

(Hoffmann, 2016, p. 368, traduzione dell'autrice)

Hoffmann osserva che questo processo di riflessione è intenzionale quando l'obiettivo esplicito della costruzione di un argomento è quello di stimolare la riflessione su di sé. Tale processo è, invece, non intenzionale quando un argomento viene costruito o ricostruito per altri scopi, come ad esempio per convincere qualcuno, ma i processi indicati nella definizione vengono comunque stimolati. A volte la riflessione su di sé può essere stimolata anche dall'ascolto di argomentazioni prodotte da altri; questo capita quando colui che propone un argomento sa coinvolgere gli ascoltatori in un confronto collaborativo, in modo che essi riescano a mettere in relazione il ragionamento proposto con il proprio. Hoffmann suggerisce tre diversi approcci attraverso i quali favorire l'attivazione di processi di argomentazione riflessiva. Il primo approccio, detto approccio semiotico, consiste nel concentrarsi su una rappresentazione delle proprie argomentazioni e valutare la qualità di queste mediante standard consolidati di buon ragionamento. Hoffmann afferma che esiste una condizione preliminare per l'argomentazione riflessiva, ovvero la possibilità di rappresentare le argomentazioni. Secondo Hoffmann, la riflessione è, infatti, possibile solo se guardiamo qualcosa, dove il termine "guardare" è inteso in senso metaforico, in quanto possiamo anche riflettere su un argomento attraverso l'ascolto.

Il secondo approccio, definito da Hoffmann approccio sociale, si concentra sulle argomentazioni, intese come dialoghi ragionati o le "controversie" tra individui. L'idea di base è che interagire con qualcuno che si oppone al proprio ragionamento, o che propone un ragionamento diverso, stimoli la riflessione su di esso e favorisca l'acquisizione di nuove prospettive sul tema oggetto di discussione. Questo approccio all'argomentazione riflessiva è in linea con l'idea di argomentazione condivisa dalla comunità di ricercatori in didattica della matematica. Stylianides, Bieda e Morselli (2016), ad esempio, sottolineano il carattere sociale dell'argomentazione, definendola come «il discorso o i mezzi retorici (non necessariamente matematici) usati da un individuo o un gruppo per convincere gli altri che un'affermazione è vera o falsa» (Stylianides et al., 2016, p. 316, traduzione dell'autrice).

Anche la definizione proposta da Baker (2003) situa l'argomentazione all'interno di un contesto di discussione, mettendo in luce come tale contesto favorisca lo sviluppo e la condivisione di significati: «Vediamo l'interazione argomentativa fondamentalmente come un tipo di gioco dialogico o dialettico che si gioca e nasce dal terreno della risoluzione collaborativa dei problemi e che è associato alla creazione collettiva dei significati» (Baker, 2003, p. 48, traduzione dell'autrice).

Il terzo approccio proposto da Hoffmann, denominato approccio guidato da modelli, si basa sull'idea che sia possibile fornire "modelli di ragionamento" agli studenti, che possano rappresentare per loro un riferimento, sia per costruire argomentazioni o processi di ragionamento che, più in generale, per riflettere su di essi e sui contenuti oggetto delle argomentazioni stesse. Questa idea consente di introdurre il terzo ed ultimo elemento che compone il quadro teorico per questo articolo, spostando l'attenzione sul ruolo chiave che il docente può svolgere nel guidare i processi riflessivi che le attività argomentative possono stimolare. 


\subsection{Il docente come modello di comportamenti ed atteggiamenti consapevoli ed efficaci}

Le riflessioni proposte nel precedente paragrafo consentono di evidenziare l'importanza di un approccio alle discussioni di classe mirato a stimolare l'esplicitazione, da parte degli allievi, dei propri processi di pensiero, nell'ottica di "rendere visibile il pensiero" (Collins, Brown \& Newmann, 1989), e di favorire l'attivazione e la condivisione di riflessioni e di processi metacognitivi. Questo suggerisce di focalizzare l'attenzione sul ruolo che il docente svolge nel progettare e gestire discussioni di classe mirate a stimolare questo tipo di processi.

Tale ruolo è stato oggetto di ricerche, che hanno consentito di delineare un costrutto teorico, quello di insegnante come Modello di Comportamenti ed Atteggiamenti Consapevoli ed Efficaci (in seguito, $\mathrm{M}-\left(\mathrm{A}_{\mathrm{CE}}\right)$, mirato ad identificare caratteristiche ed azioni che delineano il profilo di un docente che sa porsi come modello di ragionamento per i suoi studenti, stimolando allo stesso tempo riflessioni sul piano metacognitivo (Cusi \& Malara, 2016; Cusi, 2017).

II quadro che fa da sfondo a questo modello è costituito da tre principali componenti:

- le idee di Vygostkij (1978) circa l'importanza dell'interazione sociale nei processi di sviluppo del pensiero e della centralità del contributo dell'adulto o del compagno esperto nel favorire il superamento, da parte dell'allievo, del gap tra sviluppo potenziale e sviluppo attuale;

- le riflessioni di Leont'ev (1977) sul ruolo di attività svolte in contesti di socialità, non solo nel favorire la condivisione dei significati costruiti collettivamente, ma anche nel far sviluppare, da parte dell'individuo, il senso personale attribuito a tali attività e i motivi che favoriscono o meno lo sviluppo di una reale consapevolezza dei processi di apprendimento;

- il già citato modello dell'apprendistato cognitivo (Collins, Brown \& Newmann, 1989), che sottolinea l'importanza di dare agli studenti l'opportunità di osservare, scoprire o inventare le strategie degli esperti nel contesto stesso in cui vengono attivate.

Nella seguente tabella (adattata da Cusi, 2017) sono sintetizzati i ruoli che caratterizzano l'approccio di un docente che si pone come $\mathrm{M}-\mathrm{CA}_{\mathrm{CE}}$ : i ruoli e la loro caratterizzazione compaiono nella prima coIonna, mentre la seconda colonna contiene possibili indicatori per fare riferimento a tali ruoli nell'ambito dell'analisi di una discussione di classe.

I sette ruoli riassunti in Tabella 1 possono essere raggruppati in due principali gruppi. I ruoli che appartengono al primo gruppo $(a, b, c, d)$ si riferiscono a quei momenti delle discussioni di classe durante i quali il docente si pone, di fronte all'attività oggetto della discussione, non come semplice esperto, ma come learner e modello per gli studenti, esplicitando gli obiettivi nascosti, il senso delle strategie attivate, I'interpretazione dei risultati raggiunti.

I ruoli appartenenti al secondo gruppo $(e, f, g)$ si riferiscono, invece, a quei momenti delle discussioni durante i quali il docente diventa un referente per la classe nel chiarire aspetti salienti a vari livelli e nello stimolare lo sviluppo di una reale consapevolezza del senso delle attività condotte e dei processi stessi di apprendimento.

\section{Ruoli svolti da un docente che si pone come $\mathrm{M}-\mathrm{CA}_{\mathrm{CE}}$ e loro caratterizzazione}

Indicatori per l'analisi dei ruoli

(a) Soggetto che indaga e parte integrante del gruppo classe

L'insegnante cerca di stimolare nei suoi studenti un atteggiamento di ricerca nei confronti dei problemi da affrontare, chiedendo loro di fare proposte e dare suggerimenti su come procedere nell'affrontare l'attività.
Fa uso della prima persona plurale nel porre questioni mirate a chiarire che tipo di "attività di ricerca" il gruppo classe è chiamato a svolgere.

Per far sì che tutti gli allievi si sentano coinvolti come gruppo nell'attività, l'insegnante accoglie le diverse proposte senza formulare giudizi. 
L'insegnante condivide, anziché trasmettere, le strategie che adotta e le conoscenze che attiva. Lo fa cercando di porsi, di fronte all'attività da affrontare, con un atteggiamento di ricerca, con l'obiettivo costante di condividere processi di pensiero e le strategie da attivare. (b) Guida operativalstrategica

Pone domande/fa affermazioni mirate a stimolare la ricerca e di strategie di approccio ad un problema da risolvere, oppure a chiarire come un esperto potrebbe porsi quando analizza tale problema:

- Che significato ha questa domanda?

- Cosa ci viene richiesto di fare?

- Cosa potrebbe essere utile trovare per rispondere a questa domanda?

- Quali dati possono servirci?

- È I'unico modo possibile di risolvere questo problema?

\section{(c) Attivatore di processi interpretativi}

L'insegnante stimola la corretta identificazione dei frame concettuali ai quali riferirsi per interpretare e trasformare le rappresentazioni che vengono costruite. Lo fa chiedendo agli allievi di interpretare le rappresentazioni costruite nel corso della discussione e di analizzare i risultati ottenuti, facendo esplicito riferimento al contesto introdotto dal problema che viene affrontato.

\section{(d) Attivatore di pensieri anticipatori}

L'insegnante stimola l'attivazione dei corretti pensieri anticipatori per poter prevedere la forma finale (o intermedia) di una o più rappresentazioni/espressioni costruite per risolvere un problema e le trasformazioni necessarie per raggiungerla, oppure gli approcci strategici più efficaci per affrontare il problema in esame. Lo fa esplicitando spesso gli obiettivi dell'attività che si sta affrontando o i sotto-obiettivi associati alla strategia attivata, in modo che tutti gli allievi condividano tali obiettivi e facciano riferimento ad essi per monitorare le strategie attivate.

(e) Guida nel favorire un equilibrio armonico tra piano sintattico e piano semantico

L'insegnante aiuta gli studenti a controllare la correttezza sintattica e il significato delle rappresentazioni che vengono costruite.

\section{f) Guida riflessiva}

L'insegnante stimola riflessioni sugli approcci efficaci adottati durante l'attività di classe in modo che gli studenti riescano ad identificare modelli strategici ai quali ispirarsi.

- E se facessimo questa scelta, cosa potremmo ottenere?
Pone domande mirate a chiarire i significati delle rappresentazioni che vengono costruite dagli allievi e a stimolare una continua interpretazione delle rappresentazioni e dei risultati che vengono via via ottenuti nel corso della risoluzione di un problema:

- Che significato ha questo simbolo/espressione/grafico?

- Come possiamo rappresentare questa informazione?

- Che interpretazione possiamo dare di questo risultato?

- Questa soluzione è accettabile?

Pone domande/fa affermazioni mirate a far focalizzare l'attenzione sull'obiettivo (o su specifici sotto-obiettivi) dell'attività che la classe sta affrontando o della strategia adottata, oppure a far esplorare ipotesi e a far identificare gli effetti di una possibile trasformazione operata o di una possibile strategia attivata:

- Qual è l'obiettivo?

- Ricordate che l'obiettivo è...

- In questo modo raggiungiamo l'obiettivo prefissato?

- Che risultato ci aspettiamo di ottenere?

Pone domande/fa affermazioni mirate a far riflettere gli studenti sulla correttezza (o meno) delle trasformazioni che vengono eseguite sulle rappresentazioni costruite nel corso della risoluzione di un problema e su eventuali problematiche insorte in fase risolutiva, oppure a evidenziare le connessioni tra i processi che caratterizzano gli approcci adottati per risolvere il problema in esame e i corrispondenti significati:

- Questa trasformazione è corretta?

- Perché hai eseguito questa trasformazione?

- Come mai abbiamo ottenuto questo risultato?

Pone domande/fa affermazioni mirate a far attivare processi argomentativi per esplicitare il senso di una efficace strategia attivata e a far sì che gli allievi identifichino tale strategia come possibile modello di approccio al problema. Lo fa chiedendo agli studenti di esplicitare i propri processi di pensiero o di interpretare quanto affermato da altri studenti, oppure riformulando egli stesso l'approccio proposto con l'obiettivo di sottolineare le ragioni sottese a tale approccio:

- Puoi spiegare ai tuoi compagni come hai ragionato per ...? - C'è qualcuno che sa spiegare come ha ragionato ...?

- Marco ha ragionato in questo modo: "visto che voglio ottenere questo risultato, allora potrei ..." (il docente parla in prima persona, ripetendo quanto detto dall'allievo).

- Ė chiaro per tutti cos'ha detto Marco? Marco ha osservato che.... (il docente ripete quanto detto da un allievo servendosi della terza persona singolare).

Nel caso di strategie non efficaci proposte da un allievo, il docente ripete quanto detto dall'allievo per far focalizzare I'attenzione degli altri studenti su aspetti problematici delle affermazioni proposte. 
(g) Attivatore di atteggiamenti riflessivi e di atti metacognitivi

L'insegnante stimola atteggiamenti metacognitivi, con focus sul controllo del senso globale dei processi attivati.
Pone domande mirate a far sì che gli allievi esplicitino il senso delle strategie che propongono e a favorire un confronto tra diversi processi di pensiero e strategie che vengono attivati e una riflessione sull'efficacia (o meno) di specifiche scelte strategiche:

- Come mai hai scelto questa rappresentazione?

- Come mai la tua scelta è produttiva?

- Cosa ne pensate della proposta di Marco?

- Che differenze ci sono tra questa risposta e quest'altra?

Che cos'hanno in comune?

- Perché secondo voi questa strategia è efficace?

Tabella 1. I ruoli svolti da un docente che si pone come $\mathrm{M}-\mathrm{CA} \mathrm{CE}_{\mathrm{E}}$ con relativi indicatori.

Nel prossimo paragrafo (par. 4) verranno analizzati alcuni stralci tratti da una discussione condotta in una classe seconda di una scuola secondaria di primo grado, ${ }^{2}$ composta da 15 studenti. Prima di procedere con I'analisi, si introdurrà il contesto della ricerca, descrivendo la metodologia didattica adottata e presentando il problema oggetto della discussione.

\subsection{Metodologia didattica}

In linea con quanto discusso nei paragrafi nei quali è stato introdotto il quadro teorico, la progettazione e l'implementazione dell'attività che viene presentata e analizzata in questo articolo traggono ispirazione da un approccio alla valutazione formativa caratterizzato da un focus sull'argomentazione (Cusi, Morselli \& Sabena, 2017a, 2017b), nel senso di argomentazione riflessiva (Hoffmann, 2016). Nella prima parte della lezione in esame (della durata di circa 2 ore), gli studenti, suddivisi in piccoli gruppi (costituiti da 2 o 3 studenti) omogenei per competenze, lavorano su schede cartacee (si veda il par. 3.2). II ricercatore è presente in aula, assieme al docente. II docente e il ricercatore monitorano il lavoro dei gruppi, in modo da identificare possibili gruppi in difficoltà e fornire loro una scheda di aiuto (Cusi, Morselli \& Sabena, 2017a, 2017c), che sarà presentata nel prossimo paragrafo. In questa fase di monitoraggio, docente e ricercatore hanno anche modo di identificare le risposte che potranno essere condivise e discusse nella seconda parte della lezione, ipotizzando come raggrupparle e ordinarle per rendere più ricca la riflessione collettiva.

Per favorire la condivisione, i protocolli scritti prodotti da ciascun gruppo vengono fotografati in formato digitale, raggruppati e ordinati all'interno di un file .doc e successivamente mostrati attraverso la LIM.

\subsection{Il problema analizzato}

Il problema che è stato affrontato dagli studenti nel corso dell'attività si situa nel contesto dell'early algebra e costituisce un adattamento del problema "Barriera corallina", tratto dall'unità ArAl "Successioni come funzioni" (Malara, Navarra \& Sini, 2012).

Le schede di lavoro oggetto dell'attività analizzata in questo articolo fanno parte di una sequenza costituita da 4 schede di lavoro, corredate di schede di aiuto (Cusi, Morselli \& Sabena, 2017c), che delineano un percorso denominato "Sea world" ${ }^{3}$. La prima scheda e una delle schede di aiuto pre-

2. La scuola secondaria di primo grado in Italia dura tre anni e corrisponde ai primi tre anni di scuola media nel Canton Ticino. 3. Tale sequenza è stata progettata assieme ad alcuni docenti che fanno parte di un gruppo di ricerca-azione che afferisce al Dipartimento di matematica dell'Università Sapienza di Roma. Si ringrazia, in particolare, Donatella Di Girolamo, che ha sperimentato l'attività nella sua classe. 
disposte per supportare il lavoro degli studenti in difficoltà, entrambe oggetto della discussione che sarà analizzata nei prossimi paragrafi, sono presentate in Figura 1 e Figura 2.

Le schede hanno per oggetto lo studio di una particolare relazione funzionale che collega due progressioni aritmetiche che vengono messe a confronto: la progressione aritmetica dei naturali, che costituisce la sequenza dei numeri di casa delle stelle marine (si veda il diagramma in Figura 1), e un'altra progressione, costituita dai numeri pari a partire da 4, che rappresenta la sequenza dei numeri di casa dei granchi. La relazione che connette ogni elemento della prima progressione con il corrispondente elemento della seconda progressione è rappresentabile mediante un'espressione del tipo $y=a x+b$. Infatti, si può facilmente evidenziare che, se indichiamo con $x$ il numero di casa di una stella marina e con $y$ il numero di casa del granchio dirimpettaio, la relazione che lega $x$ e $y$ è rappresentabile mediante l'espressione $y=2 x+2$ (o con un'espressione ad essa equivalente).

L'intera sequenza di schede prevede l'uso di diversi registri di rappresentazione (verbale, iconico, numerico-simbolico, grafico) e l'introduzione di diversi strumenti utili per supportare processi di generalizzazione: tabelle, grafici, diagrammi, espressioni numerico-simboliche ecc.

\section{SEA WORLD}

SCHEDA 1

Alcuni biologi hanno riprodotto una barriera corallina nel parco acquatico SEA WORLD.

Per attirare l'attenzione dei visitatori hanno creato dei ripari per le stelle marine e per i granchi in questo modo:

Le stelle marine abitano in casette numerate 1, 2, 3 e così via. Le casette dei granchi sono a loro volta numerate a cominciare da 4:4,6,8,10: e così via. Ogni stella è dirimpettaia di un granchio.

Le rispettive case sono separate da una strada tracciata sul fondo della laguna dell'atollo.

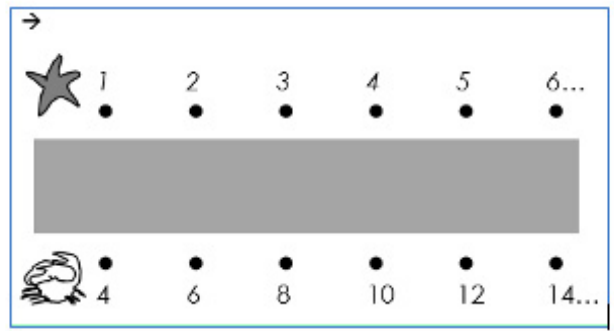

I visitatori possono partecipare ad una gara che offre la possibilità di visitare gratuitamente un'attrazione del parco a chi risponde correttamente alla seguente domanda:

Che numero di casa ha il granchio dirimpettaio della stella nella $57^{3}$ posizione?

Chi risponde alla domanda deve anche spiegare come ha ragionato, pena l'esclusione dalla gara.

Partecipa anche tu alla gara, rispondi alla domanda, spiegando come hai ragionato.

Figura 1. La prima scheda di lavoro del percorso didattico "Sea world"

In linea con l'approccio che ha ispirato il lavoro, il focus sui processi argomentativi è costante. Nella scheda 1 (Figura 1), ad esempio, viene fatta richiesta esplicita di condividere il ragionamento sviluppato per rispondere alla domanda, esplicitandolo nella propria risposta. L'ipotesi è che questa richiesta possa spingere gli studenti a non focalizzare l'attenzione soltanto sul prodotto di tale ragionamento (il numero corrispondente a 57 nella relazione, ovvero 116) e a costruire una rappresentazione che 
consenta di esplicitare il processo che può condurre a tale prodotto, come, ad esempio, un'espressione verbale, numerica o simbolica, oppure un particolare diagramma che metta in evidenza come sono stati raccolti ed interpretati i dati con l'obiettivo di attivare processi di generalizzazione.

La scheda di aiuto 1A (Figura 2) introduce una tabella per raccogliere i dati presenti nel diagramma inserito nella scheda 1, determinando ulteriori coppie di numeri corrispondenti a partire da quelli noti, e suggerisce di focalizzare l'attenzione sulle due variabili che vengono messe in relazione.

\begin{tabular}{|c|c|}
\hline \multicolumn{2}{|c|}{$\begin{array}{l}\text { AlUTO-SCHEDA } 1 \mathrm{~A} \\
\text { Costruisci una tabella per raccogliere } \mathrm{i} \text { dati relativi al numero delle casette dei granchi dirimpettai di } \\
\text { ciascuna stella. }\end{array}$} \\
\hline Numero delle casette delle stelle marine & Numero delle casette dei granchi \\
\hline 1 & \\
\hline 2 & \\
\hline 3 & \\
\hline 4 & \\
\hline 5 & \\
\hline 6 & \\
\hline 7 & \\
\hline 8 & \\
\hline 9 & \\
\hline \multicolumn{2}{|c|}{$\begin{array}{l}\text { Ora riesci a vedere che relazione c'è tra il numero delle casette delle stelle marine ed il corrispondente } \\
\text { numero delle casette dei granchi? }\end{array}$} \\
\hline
\end{tabular}

Figura 2. La scheda di aiuto a supporto della prima scheda di lavoro del percorso "Sea world".

\section{Analisi di alcuni stralci di discussione}

Come anticipato in precedenza, in questo paragrafo si proporrà l'analisi di alcuni stralci tratti dalla discussione di classe focalizzata sulle risposte fornite, dagli studenti della classe seconda coinvolta nella sperimentazione, durante il loro lavoro sulle schede 1 e 1A, presentate nel precedente paragrafo. L'obiettivo dell'analisi degli stralci di discussione, che è stata condotta dal ricercatore, è quello di evidenziare i ruoli che il docente può assumere nell'ambito di discussioni di classe mirate a realizzare processi di argomentazione riflessiva (Hoffmann, 2016) associati all'attivazione delle cinque specifiche strategie di valutazione formativa descritte nel par. 2.1 (Black \& Wiliam, 2009). Si cercherà, in particolare, di mettere in evidenza le connessioni tra i ruoli attivati (con riferimento al costrutto M-CA ${ }_{C E}$ ), le strategie di valutazione formativa e i processi di argomentazione riflessiva.

\subsection{Stralcio 1: focus sui criteri per far scaturire riflessioni su una risposta errata}

II primo stralcio si situa nella parte iniziale della discussione. II ricercatore (indicato in seguito con la lettera R) decide di mostrare alla LIM una risposta che evidenzia una errata generalizzazione da parte del gruppo di studenti che l'ha prodotta (Figura 3). La discussione inizia con un intervento di R, che ha l'obiettivo di chiarire quale sarà il tipo di approccio all'analisi delle risposte degli studenti che caratterizzerà l'intera discussione. 


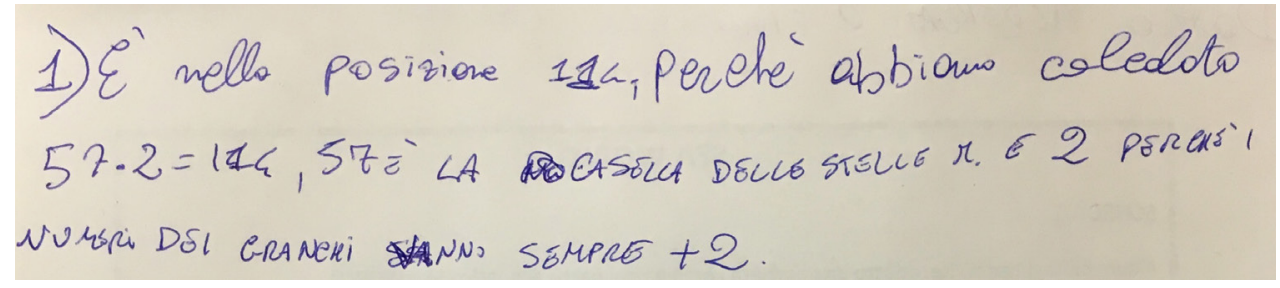

Figura 3. La prima risposta proiettata durante la discussione.

1. R: «Obiettivo di questo lavoro non sarà solo trovare la risposta corretta, ma vedere come si può ragionare per trovarla e anche vedere qual è la spiegazione più completa. Se devo spiegare il mio ragionamento, devo poterlo far capire agli altri, quelli che leggono, quindi deve essere chiaro. Poi deve essere corretto, non ci devono essere errori. Poi dev'essere completo, cioè si deve capire bene tutto ciò che porta alla risposta. Mentre analizziamo le risposte, teniamo questa cosa in mente» [Alza la mano $\mathrm{S} 1^{4}$ per intervenire].

$\mathrm{R}$ introduce la discussione ponendosi come attivatore di atteggiamenti riflessivi ed atti metacognitivi (ruolo $g$ del modello $\mathrm{M}-\mathrm{CA}_{\mathrm{CE}}$ ). L'esplicitazione iniziale (intervento 1) dei criteri che guideranno I'analisi delle risposte (correttezza, chiarezza e completezza) è in sintonia con l'idea di Hoffmann di approccio all'attivazione di processi di argomentazione riflessiva guidato da modelli, visto che i tre criteri permettono di delineare un modello di riferimento al quale ispirarsi sia per analizzare le diverse risposte che sono state fornite, sia per costruire, nel corso delle successive attività, risposte corrette, chiare e complete.

2. S1: «Non si capisce bene il ragionamento...».

3. R: «Quindi per te non è tanto chiaro il ragionamento».

4. S1: «Sì, si capisce, ma non perfettamente. Poi il numero della casella del granchio non è giusto, secondo me».

5. R: «Quindi secondo te non è del tutto chiaro quello che hanno scritto. In più, secondo te $c^{\prime}$ è un errore cioè la risposta corretta non è 114. Vorrei sapere dagli altri cosa ne pensano. Siete d'accordo con lui?»

6. S2: «Per il fatto del ragionamento, ha ragione lui».

7. R: «Come ha ragionato questo gruppo?»

8. S3: "Questo gruppo ha fatto 57, che sarebbe il numero della stella, per 2, che sarebbe il numero della stella moltiplicato per ottenere il numero della casa del granchio. Hanno moltiplicato per 2. Avrebbero dovuto fare 58 per 2 perché praticamente...».

9. R: «Prima di correggere la risposta, vediamo cosa ne pensano gli altri. Quindi anche tu pensi che la risposta corretta non sia 57 per 2?»

10. S3: «Sì».

(...) [Altri studenti dichiarano di essere d'accordo con quanto osservato da S1 ed S3].

Negli interventi 3 e 5, nei quali esplicita i criteri ai quali i commenti dello studente S1 possono essere associati, $\mathrm{R}$ continua a porsi come attivatore di atteggiamenti riflessivi ed atti metacognitivi (ruolo $\mathrm{g}$ del modello $\mathrm{M}-\mathrm{CA}_{\mathrm{CE}}$ ), con l'obiettivo di consolidare il modello delineato nell'intervento 1.

La condivisione di tali criteri, che corrisponde all'attivazione della strategia 1 di valutazione formativa, e la richiesta di fare riferimento ad essi per analizzare la risposta mostrata alla LIM fa sì che gli studenti

4. S1, S2, ... indicano i diversi studenti che intervengono nel corso della discussione. 
(interventi 2, 4, 8, 10) forniscano feedback agli autori della risposta (strategia 3), attivandosi come risorse per i compagni (strategia 4).

13. R: «Quando voglio verificare se una strategia è corretta oppure no, cosa posso fare?»

14. S4: «Possiamo fare il disegno e scrivere tutti i numeri».

15. S1: «Ë la stessa cosa che volevo dire io...cioè continuiamo la striscia dei numeri. Quelli della stella sarebbero $1,2,3,4, \ldots$ fino a 57 . Quella dei granchi sarebbe $4,6,8,10,12 \ldots$ fino al numero che sarebbe stato sotto a 57 ».

16. R: «Mi può bastare anche solo quello che c'è scritto lì [indica la scheda 1, proiettata alla LIM] per far vedere che la strategia di moltiplicare per 2 non è corretta?»

17. S5: «Loro hanno fatto 57 per 2. Ma allora, visto che 2 per 2 fa 4, al posto del 6 avrebbe dovuto esserci il 4».

(...) [R invita S5 ad avvicinarsi alla LIM per spiegare il proprio ragionamento facendo riferimento al diagramma presente nella scheda 1]

19. S5: «Se fare 57 per 2 fosse giusto, allora anche il 6 [indica il numero 6 nella successione dei numeri di casa delle stelle], moltiplicato per 2 , dovrebbe dare questo risultato [indica il numero 14 presente, in corrispondenza del 6 , nella successione dei numeri di casa dei granchi]. Ma è errato perché 6 per 2 fa 12, non 14. Quindi non è corretto fare per 2».

Quest'ultima parte del primo stralcio di discussione inizia con un intervento di R (intervento 13) mirato a fornire un feedback per supportare gli studenti nel monitoraggio del proprio lavoro. Ponendosi come guida operativa/strategica (ruolo $b$ del modello $\mathrm{M}-\mathrm{CA}_{\mathrm{CE}}$ ) e come attivatore di atteggiamenti riflessivi e di atti metacognitivi (ruolo $g$ del modello $\mathrm{M}-\left(\mathrm{A}_{\mathrm{CE}}\right.$ ), infatti, $\mathrm{R}$ mira a far sì che gli studenti identifichino ed esplicitino possibili strategie di controllo dei processi attivati. In questo modo, le strategie proposte dagli studenti (interventi $14,15,17,19$ ) diventano il nuovo oggetto della discussione. In particolare, in linea con il modello dell'apprendistato cognitivo, S5 viene invitato a chiarire le proprie osservazioni facendo riferimento al diagramma presente nella scheda 1 e mostrato alla LIM. In questo modo vengono identificati controesempi che mettono in luce la non correttezza della relazione proposta nella risposta in Figura 3.

\subsection{Stralcio 2: focus sul confronto tra risposte per stimolare l'esplicitazione dei possibili processi di generalizzazione}

Poiché tutti i gruppi di studenti, ad eccezione di quello che ha prodotto la risposta in Figura 3, hanno identificato la risposta corretta alla prima domanda presente nella scheda 1, il focus della discussione si sposta sui processi di ragionamento che hanno condotto alle risposte corrette. Inizialmente viene proposta la risposta di un gruppo che ha semplicemente costruito l'intera successione dei numeri di casa delle stelle e dei corrispondenti numeri di casa dei granchi, come suggerito anche nei due interventi 14 e 15 del precedente stralcio. $R$ guida la classe ad osservare che questo approccio risulta poco efficace perché non sarebbe attivabile nel caso in cui la domanda richiedesse di determinare il corrispondente di un numero molto grande.

Successivamente, R mostra alla LIM le risposte fornite da tre gruppi (Figura 4) che propongono la stessa rappresentazione della relazione che lega il numero di casa di una stella al corrispondente numero di casa del granchio dirimpettaio (in tutte e tre le risposte, infatti, si afferma che il corrispondente del numero di casa di una stella si ottiene moltiplicando per due il successivo del numero della stella). Nelle prime due risposte in Figura 4 (a e b), tale relazione è esplicitata soltanto a livello verbale, mentre nella terza risposta (c) viene proposto un diagramma (che verrà indicato in seguito con il termine rappresentazione sagittale) che mette in evidenza il fatto che ciascun numero della seconda progressione è il doppio del successivo del numero che gli corrisponde nella prima progressione. 
A

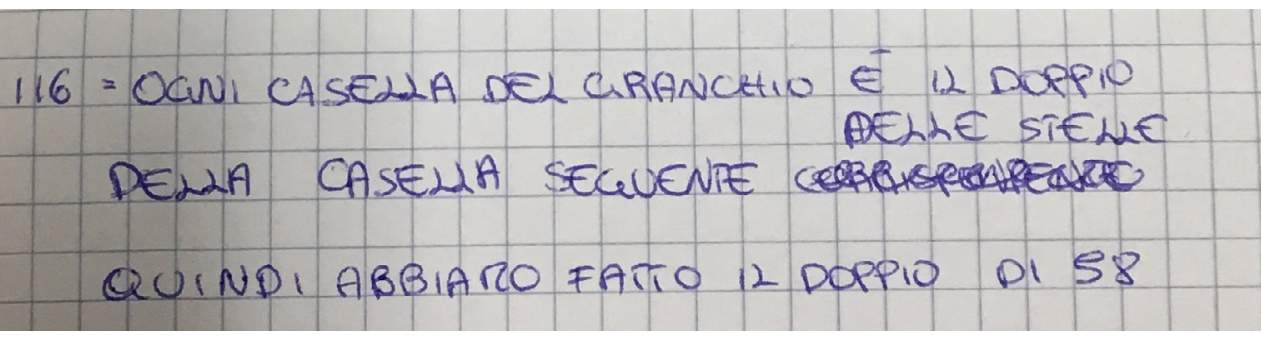

B

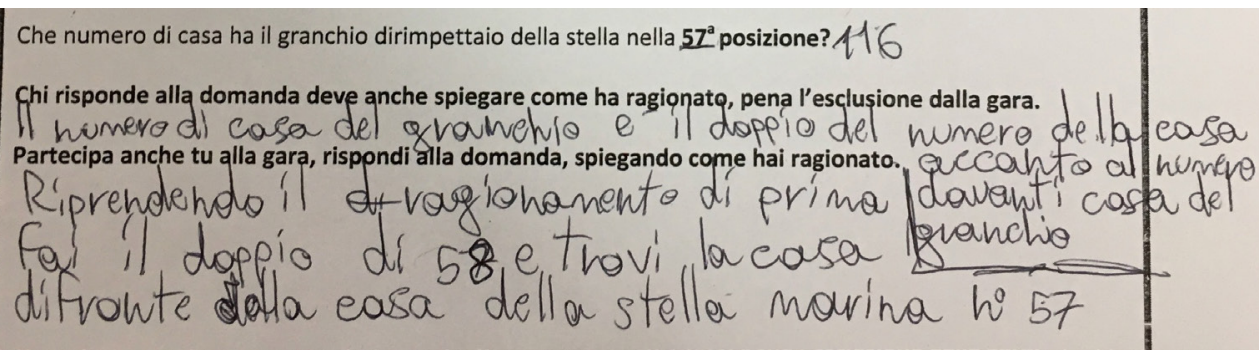

C

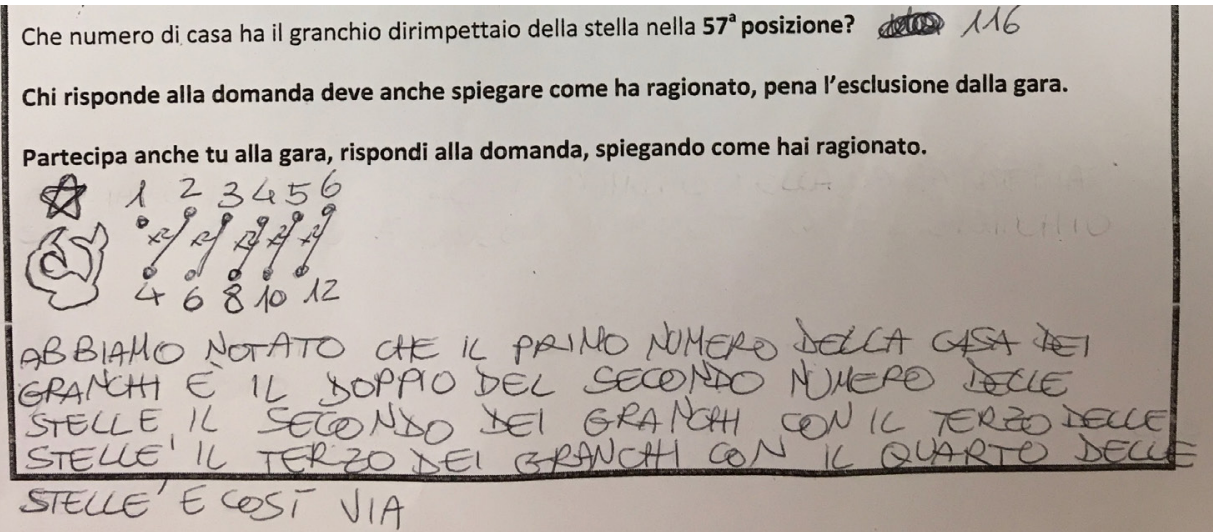

Figura 4. Le tre risposte $(a, b, c)$ che vengono messe a confronto nel secondo stralcio di discussione.

28. R: «Secondo voi perché le ho messe tutte nella stessa pagina?»

29. S6: "Secondo me perché sono simili. II ragionamento è lo stesso».

30. R: «Che ragionamento è stato fatto per trovare la risposta?»

31. S6: "Che ogni casella del granchio è il doppio della casella avanti a quella corrispondente delle stelle».

32. R: «Vieni a ripetere questo ragionamento alla LIM. Quello che adesso hai detto a parole, ripetilo guardando il disegno».

33. S6: «I numeri sulla fila del granchio sono il doppio dei numeri della fila della stella marina, ma non di quella corrispondente, ma di quella avanti a quella corrispondente. Ad esempio, 4 è il doppio di 2, 6 è il doppio di 3, 8 è il doppio di 4... [Indica i vari numeri nel diagramma]».

34. R: "Se volessi riassumere il metodo usato da questi tre gruppi, che cosa potrei scrivere in simboli matematici?»

(...) [Al termine del momento di confronto, la classe concorda che l'espressione che meglio rappresenta il ragionamento proposto nelle prime tre risposte è $(57+1) \cdot 2]$

Il primo intervento di R (intervento 28) pone la questione ancora sul piano metacognitivo, visto che si pone come attivatore di processi riflessivi e di atti metacognitivi (ruolo $g$ del modello $\mathrm{M}-\mathrm{CA}_{\mathrm{CE}}$ ), 
chiedendo agli studenti di identificare i criteri che hanno guidato la sua raccolta delle risposte. Leggere le tre risposte con l'obiettivo di ipotizzare le motivazioni alla base della scelta di inserirle nello stesso gruppo fa sì che gli studenti focalizzino l'attenzione sugli elementi comuni a tali risposte, identificando così la struttura che caratterizza il processo di generalizzazione sotteso. Questo situa I'approccio alla reflective argumentation, adottato da $\mathrm{R}$ in questo stralcio di discussione, in un ambito semiotico (secondo la terminologia di Hoffmann), visto che le rappresentazioni verbali e iconiche dei processi di ragionamento attivati dai gruppi di studenti che hanno fornito le tre risposte diventano il principale oggetto di analisi e riflessione. II focus su un approccio semiotico è ancora più evidente nell'ultimo intervento di $R$ inserito in questo stralcio (intervento 34). Infatti, $R$ si pone da attivatore di processi interpretativi (ruolo $\mathrm{c}$ del modello $\mathrm{M}-\mathrm{CA}_{\mathrm{CE}}$ ), stimolando gli studenti a costruire una nuova rappresentazione (numerico-simbolica) della relazione espressa nelle tre risposte. Questa richiesta ha I'obiettivo di supportare il confronto tra questa rappresentazione e quelle associabili ad altre risposte che successivamente verranno discusse, esplicitando gli elementi che caratterizzano i processi di generalizzazione attivati dai diversi gruppi e stimolando la ricerca delle ragioni alla base dell'equivalenza tra le diverse espressioni costruite nel corso della discussione.

Notiamo che anche in questo stralcio R svolge un importante ruolo mirato a far attivare gli studenti come risorse gli uni per gli altri (strategia 4), ovvero quello di guida riflessiva (ruolo $f$ del modello $\mathrm{M}-\left(\mathrm{A}_{\mathrm{CE}}\right.$ ), poiché invita $\mathrm{S} 6$ (intervento 32 ) ad esplicitare le proprie osservazioni facendo riferimento al diagramma a disposizione, quindi appoggiandosi ad un'altra rappresentazione.

\subsection{Stralcio 3: riflessioni meta sull'uso di specifici strumenti a supporto del ragionamento}

Il terzo stralcio riguarda la fase della discussione in cui i gruppi che hanno utilizzato la scheda di aiuto $1 \mathrm{~A}$ (Figura 2) vengono invitati a riflettere sull'efficacia dell'aiuto utilizzato e sui processi di ragionamento che il supporto fornito ha permesso di stimolare.

$\mathrm{R}$ mostra la risposta (Figura 5) di una coppia che ha ricevuto l'aiuto della scheda 1A. Inizialmente il focus della discussione è sul ragionamento che il gruppo ha condotto. La classe, stimolata ancora una volta a costruire una rappresentazione che riassuma tale processo, conclude che il processo di generalizzazione sotteso può essere sintetizzato mediante l'espressione $57 \cdot 2+2$. Tale espressione viene messa a confronto con quella identificata analizzando le risposte in Figura $4[(57+1) \cdot 2]$ e viene richiesto agli studenti di esplicitare, facendo riferimento ad aspetti sintattici (focus sulla proprietà distributiva), perché le due espressioni sono equivalenti. R, ponendosi come guida operativa/strategica, guida anche la classe a riflettere su come lo strumento rappresentazione sagittale possa supportare la costruzione di entrambe le espressioni identificate nel corso della discussione.

In un secondo momento, $\mathrm{R}$ invita gli studenti a riflettere su ciò che è scritto nella parte alta del protocollo in Figura 5: "Tra 1 e 4 ci sono 3 differenze, tra 2 e 6 [ci sono] 4 [differenze], tra 3 e 8 [ci sono] 5 [differenze], e così via. Anche la differenza tra i due numeri avanza sempre di uno, mentre nella parte del granchio avanza di 2». Questo stralcio evidenzia che il gruppo che ha prodotto questa risposta ha focalizzato inizialmente l'attenzione sulle differenze tra il numero di casa dei granchi e i corrispondenti numeri di casa delle stelle, osservando che tali differenze sono in progressione aritmetica di ragione 1, senza però esplicitare se esistono connessioni tra queste osservazioni e l'approccio successivamente adottato per rispondere alla domanda. Nel corso della discussione, gli studenti del gruppo dichiarano che tali connessioni non sono state esplicitate perché il gruppo ha abbandonato la strada tracciata inizialmente. 


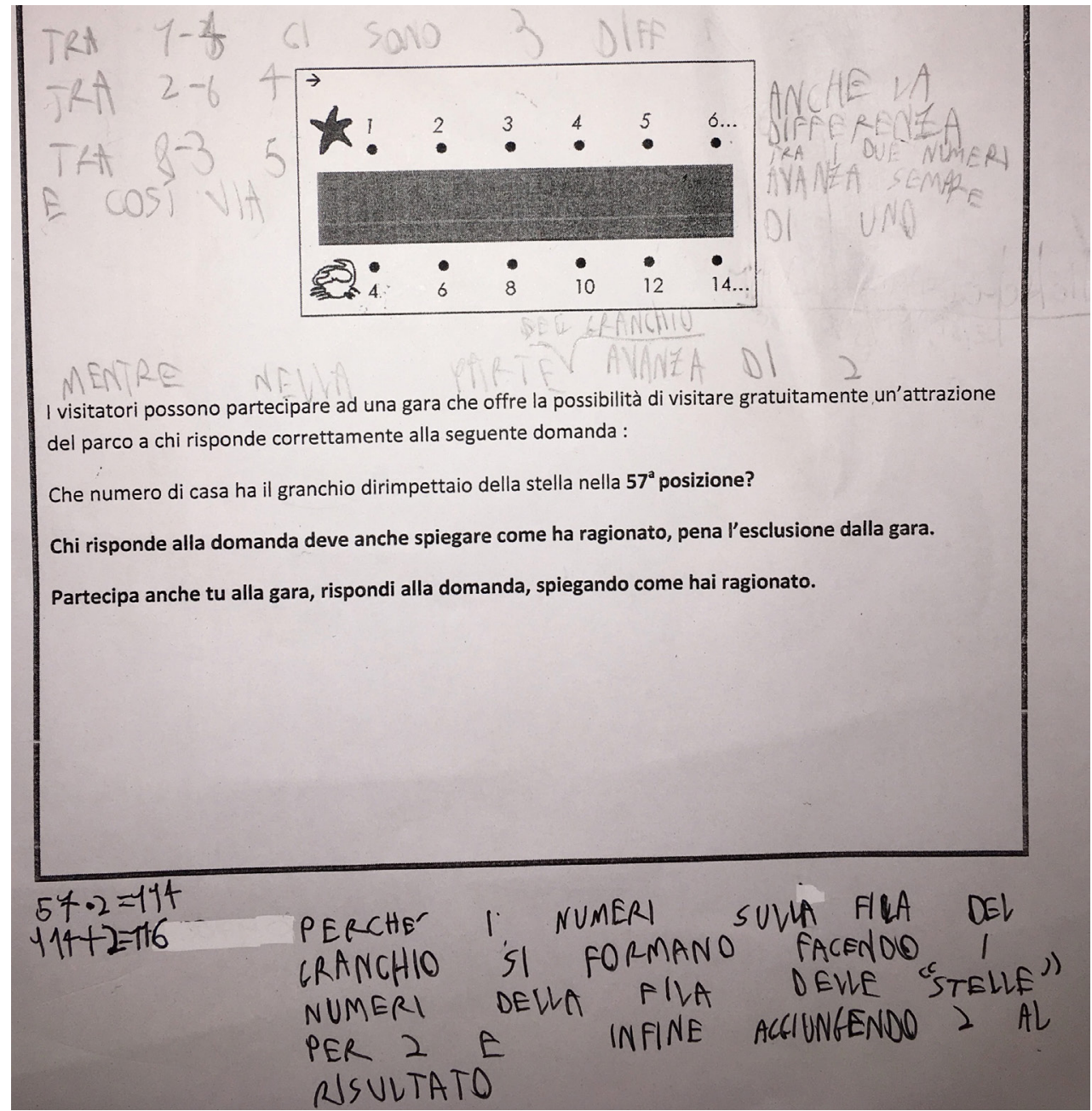

Figura 5. II protocollo prodotto dal primo gruppo di studenti che ha ricevuto l'aiuto $1 \mathrm{~A}$

Lo stralcio che viene presentato in questo paragrafo ha inizio con l'intervento di S7, componente del secondo gruppo che ha utilizzato la scheda di aiuto 1A, la cui risposta è presentata in Figura 6.

61. S7: «Anche noi abbiamo fatto questo ragionamento».

62. R: «Infatti mi voglio collegare anche al vostro ragionamento. Cosa avete osservato? Venite alla lavagna a raccontarlo... Ė un ragionamento che loro [gli studenti che hanno prodotto la risposta in Figura 5] hanno iniziato a fare e loro [gli studenti che hanno prodotto la risposta in Figura 6] hanno completato» [R mostra la risposta del gruppo di cui $\mathrm{S} 7 \mathrm{fa}$ parte, Figura 6]. 


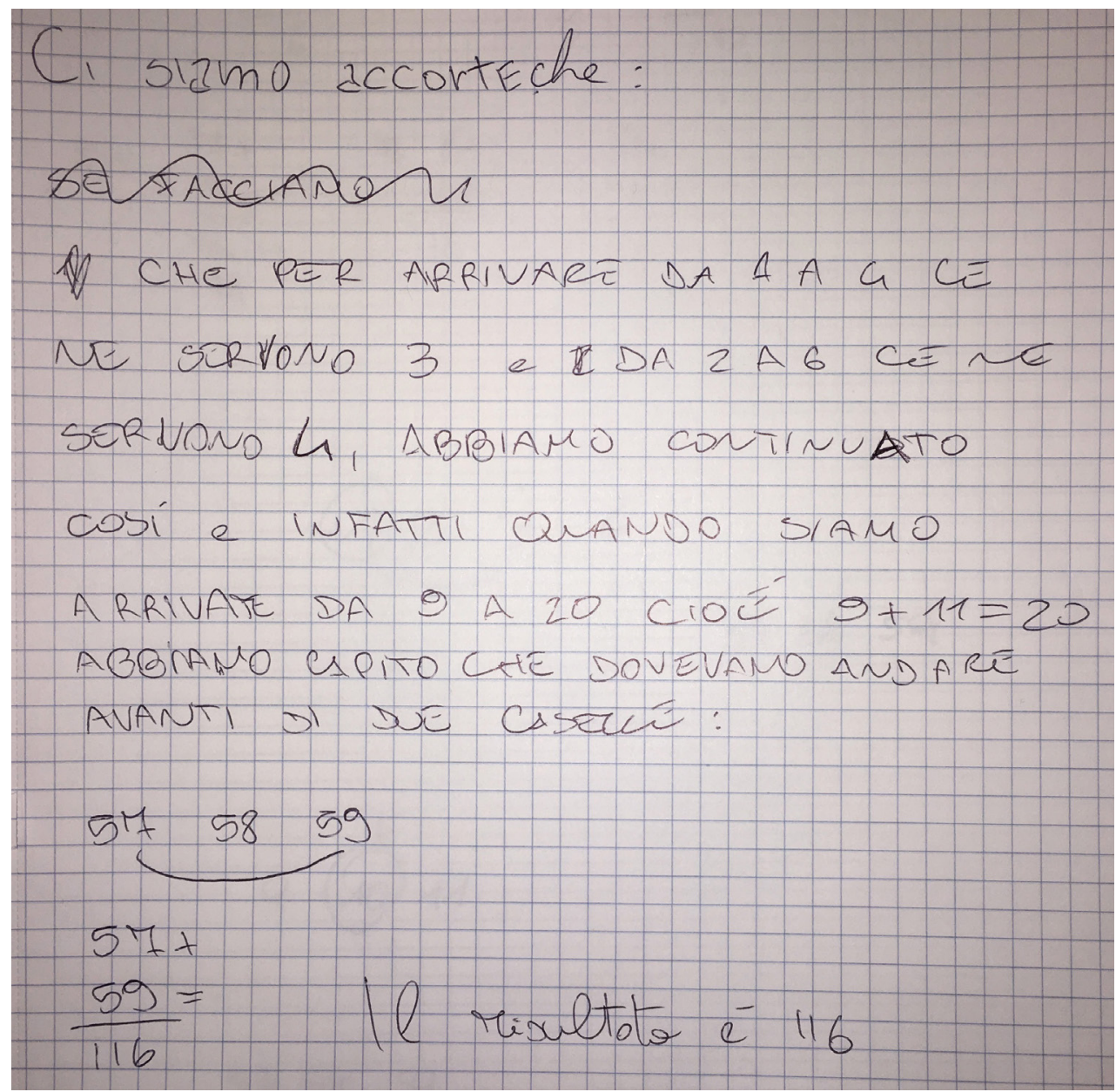

Figura 6. II protocollo prodotto dal secondo gruppo di studenti che ha ricevuto l'aiuto 1A.

L'inizio di questo stralcio mette in evidenza come l'approccio adottato stimoli gli studenti ad assumere la responsabilità del proprio apprendimento (strategia 5). Infatti, S7 identifica il ragionamento abbozzato nella parte alta di Figura 5 con il proprio (intervento 61), spostando l'attenzione sulla risposta costruita dagli studenti del suo gruppo e divenendo così protagonista dello stralcio di discussione. $\mathrm{R}$ supporta l'entrata in scena di S7, mostrando la risposta prodotta dal gruppo al quale S7 appartiene e dichiarando che il ragionamento introdotto nella parte alta della risposta in Figura 5 è stato completato da questo gruppo.

63. S7: "All'inizio non avevamo capito bene, poi la prof ci ha dato la scheda di aiuto».

64. R: «Faccio vedere a tutti la scheda di aiuto, perché non I'hanno ricevuta tutti. Poi torniamo qua» [Viene mostrata la scheda di aiuto $1 \mathrm{~A}$ ].

65. S7: "Abbiamo capito che dalla casella 1 alla casella 4 c'erano tre numeri, dalla casella 2 alla casella 6 c'erano 4 numeri, e così via... la scheda d'aiuto arrivava fino a 9, che va a 20... Quando abbiamo ottenuto tutti i risultati, abbiamo capito che ogni numero, cioè, ad esempio, 57, avanzava sempre di un numero in più, quindi abbiamo preso $57,58,59$, quindi abbiamo preso $57+59$, che fa $116 »$.

66. R: «Hanno notato che ogni volta, su questa freccia, aggiungo un numero in più...però non posso mica mettermi a disegnarli tutti!» [R ricostruisce la generalizzazione che questo gruppo ha fatto a partire dall'esplorazione numerica, costruendo il diagramma sagittale in Figura 7] 


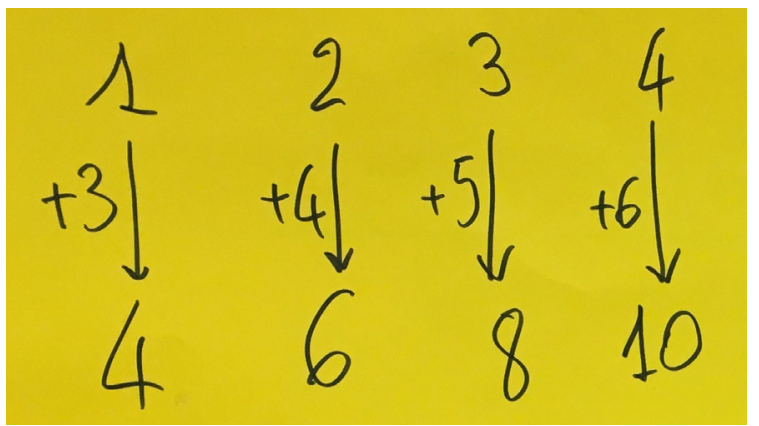

Figura 7. Immagine del diagramma sagittale costruito da R mentre esplicita (intervento 66) la prima parte del ragionamento proposto da S7.

67. R: «Potete spiegare questa parte qua della vostra risposta? [R indica lo stralcio di risposta che evidenzia la seconda parte del ragionamento proposto dal gruppo e non ancora ben esplicitato: "Quando siamo arrivate da 9 a 20, cioè $9+11=20$, abbiamo capito che dovevamo andare avanti di due caselle"] Loro hanno detto "non li andiamo a fare tutti tutti" [Si riferisce alla ricostruzione delle due sequenze di numeri fino alla coppia 57-116]».

68. S7: "Quando siamo arrivati da 9 a 20, che 9+11 fa 20, abbiamo capito che dovevamo andare avanti di 2 caselle. Quindi abbiamo scritto 57, 58, 59 e abbiamo preso 59, quindi $57+59$, che fa 116» [Mentre S7 spiega il suo ragionamento, R completa la rappresentazione sagittale precedentemente introdotta, Figura 8]

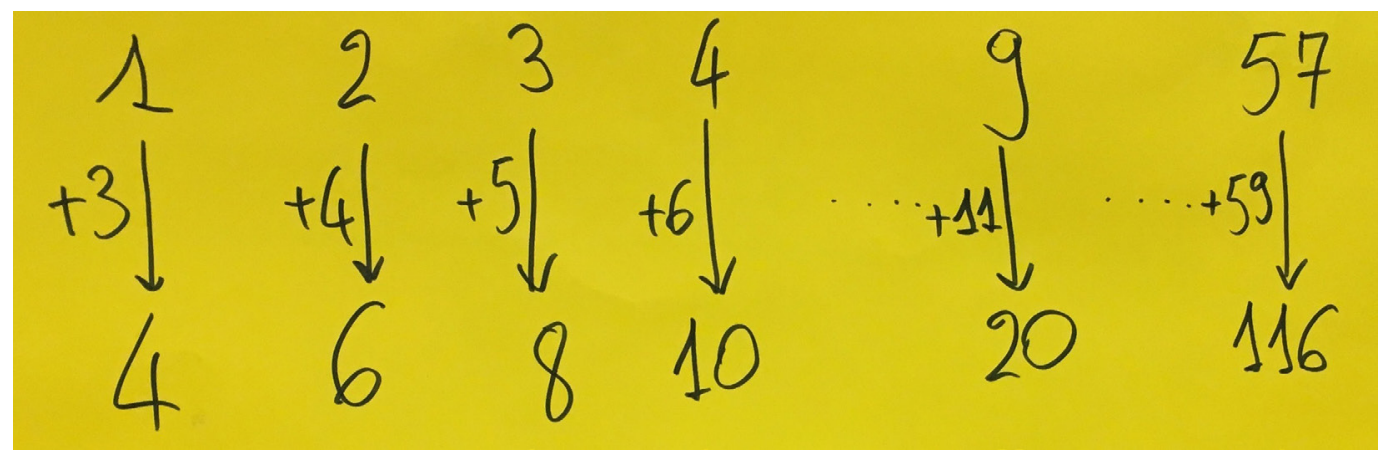

Figura 8. Immagine del diagramma sagittale completato da R per rappresentare l'intero ragionamento proposto da S7.

Negli interventi di questo stralcio di cui è protagonista (interventi 63, 65 e 68), S7 riconosce e condivide le difficoltà incontrate inizialmente e cerca di esplicitare il ragionamento che ha condotto, lei e gli altri membri del suo gruppo, ad identificare la risposta alla domanda presente nella scheda 1. Per favorire la condivisione di questi processi di ragionamento e generalizzazione, $\mathrm{R}$ si pone come guida operativa-strategica (ruolo $b$ del modello $\mathrm{M}-\mathrm{CA}_{\mathrm{CE}}$ ), esplicitando l'approccio strategico che ha condotto il gruppo a cercare di sfruttare l'osservazione sulle differenze tra numeri corrispondenti nelle due successioni per determinare il corrispondente di 57 (intervento 66) e focalizzando l'attenzione su una parte della risposta prodotta dal gruppo (intervento 67), nella quale le studentesse identificano in maniera esplicita il momento chiave in cui il processo di generalizzazione da esse attivato è stato completato. Contemporaneamente, $\mathrm{R}$ si pone come guida riflessiva (ruolo $f$ del modello $\mathrm{M}-\mathrm{CA}_{\mathrm{CE}}$ ), costruendo il diagramma sagittale (Figure 7 e 8) per rappresentare il processo di ragionamento che $\mathrm{S} 7$ sta provando ad esplicitare. Ancora una volta notiamo la messa in atto di un approccio semiotico ai processi di argomentazione riflessiva attivati nel corso della discussione. II diagramma sagittale rappresenta, infatti, lo strumento di rappresentazione che consente di rendere visibile il processo 
di pensiero attivato dagli studenti e verbalizzato nella risposta in Figura 6. Contemporaneamente, la costruzione condivisa di diagrammi sagittali in diversi momenti della discussione e l'esplicitazione delle modalità attraverso le quali interpretarli fa sì che tali diagrammi possano diventare riferimenti importanti per gli studenti, modelli ai quali ispirarsi per costruire rappresentazioni dei propri processi di pensiero e di quelli altrui per favorire il confronto e la riflessione che caratterizzano i processi di argomentazione riflessiva.

69. R: «C'è qualcuno che vuole ripetere questo ragionamento?»

70. S8: "Loro hanno capito che nella casella della stella i numeri vanno regolarmente, mentre nella casella del granchio vanno di +2 . Da 1 a 4 si aggiunge 3. Da 2 a 6, 4. Da 3 a 8, 5 . Da 4 a 10, 6. E così via... fino ad arrivare a 9+11. Loro hanno fatto 57+59, che fa 116».

71. R: «Quindi...Loro cos'hanno osservato? Hanno cercato il legame tra il numero da cui si parte e quello che c'è sulla freccia. Perché? Perché, se capisco questo legame, posso generalizzare e non ho bisogno di costruire tutte le frecce [fino a 57]».

In questa ultima parte del terzo stralcio, $\mathrm{R}$ si pone nuovamente come guida riflessiva (ruolo $f$ del modello $\mathrm{M}-\left(\mathrm{A}_{\mathrm{CE}}\right)$, chiedendo ad altri studenti di provare ad esplicitare il ragionamento proposto da $\mathrm{S} 7$ (intervento 69) ed esplicitando lei stessa l'approccio strategico che ha guidato i processi di pensiero attivati dal gruppo al quale S7 appartiene (intervento 70), parlando in prima persona per porsi anche come soggetto che indaga (ruolo a del modello $\mathrm{M}-\left(\mathrm{A}_{\mathrm{CE}}\right.$ ). Questi interventi sono mirati a far sì che gli altri studenti identifichino questo approccio come modello al quale ispirarsi quando dovranno affrontare altre attività caratterizzate dalla necessità di attivare processi di generalizzazione.

\section{Discussionederisistlat}

L'analisi presentata nel precedente paragrafo ha consentito di evidenziare sinergiche combinazioni tra specifici ruoli identificati dal modello $\mathrm{M}-\mathrm{CA}_{\mathrm{CE}}$, corrispondenti approcci ai processi di argomentazione riflessiva e strategie di valutazione formativa che vengono attivate grazie ad essi. In quest'ultimo paragrafo vengono forniti possibili spunti per la progettazione di discussioni di classe, attraverso l'esplicitazione di tali sinergie. In particolare, si evidenziano, in riferimento a ciascuno stralcio, quindi a specifici focus delle discussioni, i principali ruoli che colui che coordina le discussioni di classe può cercare di assumere, con l'obiettivo di attivare approcci diversi all'argomentazione riflessiva e realizzare processi di valutazione formativa.

L'analisi del primo stralcio ha permesso di mettere in luce come l'assunzione del ruolo di attivatore di processi riflessivi e di atti metacognitivi da parte del docente, nell'ambito di discussioni focalizzate sull'analisi delle risposte degli studenti e su eventuali errori che possono essere evidenziati, possa promuovere la condivisione di un modello di riferimento (nel caso di questa discussione, i criteri di correttezza, chiarezza e completezza) per guidare sia l'analisi delle risposte oggetto della discussione che i conseguenti processi di argomentazione riflessiva che vengono realizzati. L'attivazione di questo ruolo consente di realizzare la strategia 1 di valutazione formativa (visto che i criteri di valutazione vengono introdotti e condivisi) e, come diretta conseguenza, anche le strategie 3 e 4, poiché gli studenti diventano risorse gli uni per gli altri, fornendo feedback agli autori della risposta analizzata. Questo stralcio evidenzia anche che l'attivazione dell'ulteriore ruolo di guida operativa/strategica consente di stimolare la riflessione su strategie metacognitive per il controllo della correttezza o meno dei processi di ragionamento attivati.

Il secondo stralcio evidenzia come un approccio semiotico ai processi di argomentazione riflessiva 
possa essere realizzato attraverso la combinazione tra il ruolo di attivatore di atteggiamenti riflessivi e di atti metacognitivi, mirato a supportare il confronto e la riflessione sulle risposte prodotte dagli studenti, e quello di attivatore di processi interpretativi, attraverso il quale è possibile stimolare l'interpretazione delle rappresentazioni dei processi di ragionamento che caratterizzano le risposte degli studenti e la costruzione di nuove rappresentazioni mirate ad esplicitare tali processi. L'analisi di questo stralcio ha consentito, inoltre, di mettere in luce come la riflessione sulle diverse rappresentazioni esaminate e costruite faccia sì che gli studenti, nel momento in cui esplicitano i processi di ragionamento sottesi, si attivino come risorse gli uni per gli altri (strategia 4).

Grazie all'analisi del terzo stralcio, infine, è stato possibile evidenziare come la combinazione efficace dei ruoli di guida operativa-strategica e di guida riflessiva, nel condurre una discussione sugli strumenti forniti agli studenti per supportare i processi di ragionamento e generalizzazione, abbia favorito la presa in carico del proprio apprendimento da parte degli studenti stessi (strategia 5 di valutazione formativa). Lo studente che interviene, infatti, sceglie spontaneamente di condividere difficoltà incontrate e processi di pensiero attivati. L'introduzione del diagramma sagittale e l'esplicitazione dei significati associati a tale diagramma consentono agli studenti di identificare un'ulteriore rappresentazione del ragionamento sotteso alla risposta che viene analizzata. In questo modo, tale ragionamento viene reso visibile e, di conseguenza, realmente condiviso nel corso della discussione. Questo stralcio mette perciò in evidenza come la combinazione di questi ruoli possa stimolare un duplice approccio ai processi di argomentazione riflessiva: I'approccio semiotico, poiché il focus è ancora sulla costruzione e interpretazione di diverse rappresentazioni delle argomentazioni e dei ragionamenti proposti; I'approccio guidato da modelli, visto che il docente si pone come modello di ragionamento e fa sì che siano identificati altri efficaci modelli di ragionamento nel corso della discussione.

Osserviamo, infine, come l'approccio sociale ai processi di argomentazione riflessiva permei l'intera discussione e come questo approccio risulti il frutto di una progettazione della discussione stessa (strategia 2 di valutazione formativa) realizzata consapevolmente con l'obiettivo di stimolare un dibattito collettivo che consenta agli studenti di confrontare argomentazioni, strategie, processi di ragionamento (propri e altrui), rendendoli tangibili oggetti di riflessione.

\section{Bibliografia}

Baker, M. (2003). Computer-mediated interactions for the co-elaboration of scientific notions. In J. Andriessen, M. Baker \& D. Suthers (Eds.), Arguing to Learn: confronting cognitions in computer supported collaborative learning environments. Computer-Supported Collaborative Learning book series, vol. 1 (pp. 47-78). Dordrecht, The Netherlands: Kluwer.

Bernholt, S., Ronnebeck, S., Ropohl, M., Koller, O., \& Parchmann, I. (2013). ASSIST ME. Report on current state of the art in formative and summative assessment in IBE in STM. ASSIST-ME Report Series Number 1-2.

Black, P., \& Wiliam, D. (2009). Developing the theory of formative assessment. Educational Assessment, Evaluation and Accountability, 21(1), 5-31.

Collins, A., Brown, J. S., \& Newman, S. E. (1989). Cognitive Apprenticeship: Teaching the Crafts of Reading, Writing and Mathematics! In L. B. Resnick (Ed.), Knowing, Learning, and Instruction: Essays in Honor of Robert Glaser (pp. 453-494). Hillsdale, NJ: Lawrence Erlbaum Associates.

Cusi, A. (2017). II ruolo dell'insegnante nell'ambito di una didattica dell'algebra come strumento per ragionare: lenti teoriche per I'analisi. L'insegnamento della Matematica e delle Scienze Integrate, 40 A-B (2), 157-180.

Cusi, A., \& Malara, N. A. (2016). The Intertwining of Theory and Practice: Influences on Ways of Teaching and 
Teachers' Education. In L. English \& D. Kirshner (Eds.), Handbook of International Research in Mathematics Education 3rd Edition (pp. 504-522). Taylor \& Francis.

Cusi, A., Morselli, F., \& Sabena, C. (2017a). Promoting formative assessment in a connected classroom environment: design and implementation of digital resources. ZDM Mathematics Education, 49(5), 755-767.

Cusi, A., Morselli, F., \& Sabena, C. (2017b). Promuovere strategie di valutazione formativa in Matematica con le nuove tecnologie: I'esperienza del progetto FaSMEd. Annali online della Didattica e della Formazione Docente, 9(14), 91-107.

Cusi, A., Morselli, F., \& Sabena, C. (2017c). Designing and analysing the role of digital resources in supporting formative assessment processes in the classroom: The helping worksheets. In T. Dooley \& G. Gueudet (Eds.), Proceedings of Cerme 10 (pp. 3452-3459). Dublin, Ireland: DCU Institute of Education and ERME.

De Luca, M., Demartini, L., Migliano, P., Savioli, K., Serratore, E., \& Vio, E. (Eds.) (2008). Argomentare: un "laboratorio" per le competenze. AVIMES-VALMAT.

Ferretti, F., Paraskevi, M.-C., \& Vannini, I. (2018). Formative assessment for mathematics teaching and learning. Teacher professional development research by video analysis methodologies. Franco Angeli editore.

Hoffmann, M. H. G. (2016). Reflective argumentation: A Cognitive Function of Arguing. Argumentation, 30(4), 365-397.

Leont'ev, A. N. (1977). Attività, coscienza e personalità. Ed. Giunti Barbera.

Malara, N. A., Navarra, G., \& Sini, S. (2012). Unità 12: Successioni come funzioni. Loro esplorazioni attraverso differenti registri di rappresentazione. Progetto ArAl. Pitagora Editrice.

Radford, L., \& Demers, S. (2006). Comunicazione e apprendimento. Riferimenti concettuali e pratici per le ore di matematica. Bologna: Pitagora Editrice.

Stylianides, A. J., Bieda, K. N., \& Morselli, F. (2016). Proof and argumentation in mathematics education research. In A. Gutiérrez, G. C. Leder \& P. Boero (Eds.), The Second Handbook of Research on the Psychology of Mathematics Education (pp. 315-351). Rotterdam, The Netherlands: Sense Publishers.

Vygostkij, L. S. (1978). Mind in society: The development of higher mental processes. Cambridge, MA: Harvard University Press. 